\title{
V(Z) OF THE SURFACE ACOUSTIC WAVE FOCUSING SYSTEM
}

\author{
A. ATALAR and H. KÖYMEN
}

Electrical and Electronics Engineering Department, Middle East Technical University, 06531 Ankara, TURKEY.

\section{ABSTRACT}

It is possible to define a $V(Z)$ function for the surface acoustic wave (SAW) focusing system for a class of reflectors similar to that defined in acoustic microscopy. In this case $V(Z)$ is a function which relates the transducer output voltage to the distance between the focal point and the reflection line. It is possible to express $V(Z)$ in an analytical expression using angular spectrum techniques. $V(Z)$ is measured for straight edge reflectors forming various angles with the surface. $V(Z)$ of straight step reflectors are investigated. These curves show a dependence on the type of reflector. The experiments are performed at $1.5 \mathrm{MHz}$ in conjunction with a computerized data acquisition system. The phase information is also recorded in all these measurements in addition to the amplitude. It is possible to get an inversion of $V(z)$ to get the SAW reflection coefficient at a straight discontinuity.

\section{Introduction}

Acoustic microscopy is becoming a powerful technique in characterization and imaging in material science, biology and thin film technology [1]. It produces acoustic images of plane surfaces of materials by a spherically converging bulk wave obtained by an acoustic lens in the form of a spherical cavity. Images are sensitive to acoustic parameters of the surface material as well as to the thicknesses of the layers close to the surface. Quantitative results about the material parameters can be extracted from the information conveyed by the acoustic microscope through the measurement of what is known as $V(Z)$ curves [2], [3]. These curves are the plots of transducer output voltage, $v$, as a function of lens to object distance, $Z$. They exhibit a very strong dependence on acoustic parameters and layer thicknesses of the object material. The principal mechanism for material dependence of $V(Z)$ is the interference of nonspecular leaky surface acoustic waves (SAW) with specularly reflected bulk waves [4].

Diffraction limited SAW focusing has been successfully achieved using 'surface acoustic wave focusing axicong, (SAWFAX) [5].
In this method almost all the power available from the transducer is converted into the SAW for high impedance materials. A detailed analysis of SAWFAX may be found elsewhere [6]. SAWFAX has the potential of providing a new mode of imaging for acoustic microscopes with increased subsurface sensitivity. In this paper we present the $V(Z)$ techniques applied to this method. Theoretical $V(Z)$ formulas and the results of the experimental $V(Z)$ measurements are given. It is also shown that the SAW reflection coefficient of various straight discontinuities can be extracted from $V(Z)$ data only with limited success.

\section{Theory}

$V(Z)$ for a bulk wave focusing acoustic microscope lens has been extensively discussed in the literature. In the SAWFAX, the focused acoustic energy is in the form of SAW as opposed to a bulk wave, and the reflecting planar surface is replaced by a straight discontinuity. In this case, $V(Z)$ shows the variation of the output voltage as a function of the distance between the reflecting straight discontinuity and the SAW focus. In the following derivation we use an angular spectrum approach very similar to that used in derivation of $V(Z)$ formula for the regular acoustic microscope lens [7].

In order to find the surface wave field at the focus of the SAWFAX, we consider the geometry drawn in Fig.1. The bulk wave incident at $r-s$ plane is represented by $u+(r, s)$. The surface wave field along $x$ axis and propagating in $+z$ direction, $u_{f}+(x)$, can be written as:

$u_{f}+(x)=\int_{-\infty}^{+\infty}\left[\int_{-\infty}^{+\infty} u+(r, s) P(r, s) d s\right]$

where $k g$ is real part of the wavenumber of surface waves and $P(r, s)$ is the pupil function. Eq.1 expresses the field at the focus as the Fourier transform of the integrated incident field, because the cylindrical parabolic mirror acts like a Fourier transform operator in one dimension [8], and bulk to surface wave conversion 


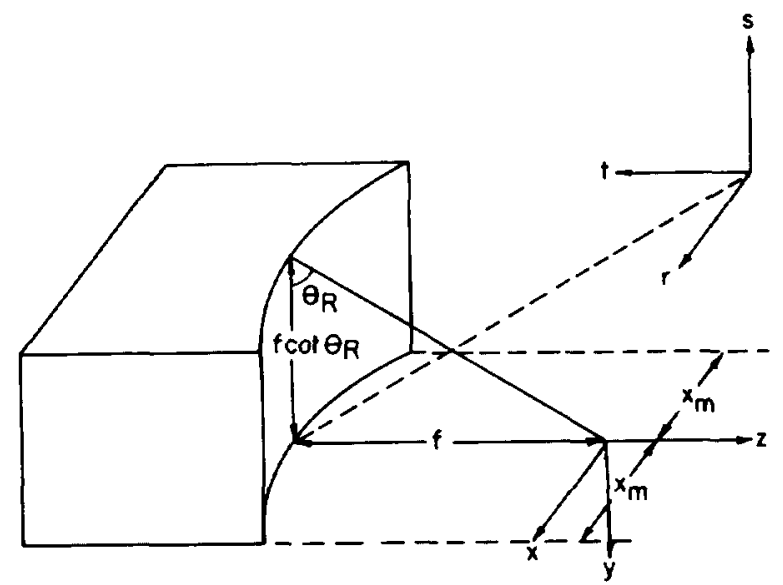

Fig.1 Geometry for $V(Z)$ calculation.

phenomenon as an integrator in the other dimension [9]. $P(r, s)$ may be given by

$P(r, s) a\left[\begin{array}{cc}\left\{\exp \left[-\left(\alpha_{D}+\alpha_{L}\right)\left(f-s \tan \theta_{R}\right)\right]\right. & -x_{R}<r<x_{R} \\ \left.\cdot \exp \left[-j k_{R} S\right]\right\} & 0<s<f \cot \theta_{R} \\ 0 & \text { otherwise }\end{array}\right.$

The pupil function here is found assuming a mirror with rectangular cross section, width of $2 x$ and a height of $f \cot \theta_{R}$. The first exponential is due to the leaky nature of SAW, and the second exponential is a linear phase term arising from the inclination of incident wavefront with respect to $r-s$ plane.

Converselj, if the surface wave field along the $x$ axis propagating in $-z$ direction, $u_{f}-$ $(x)$, is known, one can calculate the field distribution $u-(r, s)$ at $r-s$ plane as

$u-(r, s)=\alpha_{L} P(r, s) \int_{-\infty}^{+\infty} u_{f}-(x) \exp \left[-j\left(k_{R} / f\right) r x\right] d x$

To take care of the reflection process which may be angle dependent, the fields must be transformed into the angular spectrum domain. The angular spectrum of $u_{f}(x)$ is represented by $U_{f}\left(k_{x}\right)$ :

$$
U_{f}+\left(k_{x}\right)=F\left\{u_{f}+(x)\right\}
$$

Combining Eq. (4) with Eq.(1) we find

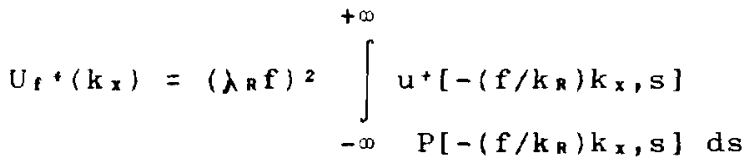

In angular spectrum domain the propagation is easily taken into account by multiplication of the $\exp \left[j \mathrm{kzz}_{\mathrm{z}}\right]$ factor where $k_{z}=\left(k_{R}^{2}+k_{x^{2}}\right)^{1} 1 / 2$ is the component of $k$ vector in $z$ direction. Due to the leaky nature of surface waves an additional exponential decay factor must be included in propagation equation. If a discontinuity with angle dependent reflection coefficient $R\left(\sin \theta_{R}\right)$ is present at plane $z=Z$, one can write the reflected spectrum, $U_{f}-\left(k_{x}\right)$ at plane $z=0$ as

$$
\begin{aligned}
U_{f}-\left(k_{x}\right)= & U_{f}+\left(k_{x}\right) \exp \left[j 2 k_{z} Z\right] \\
& \exp \left[-2\left(\alpha_{y}+\alpha_{L}\right) k_{z} Z / k_{k}\right] R\left(k_{x} / k_{k}\right)
\end{aligned}
$$

where $U_{f}^{-}=F\left\{u_{f}^{-}\right\}$

Using Eq. 7 and Eq. 3 together, one can write the field at $r-s$ plane, $u-(r, s)$ :

$u^{-}(r, s)$ 口 $\alpha_{L} \quad P(r, s) U_{k}-\left[\left(k_{R} / f\right) r\right]$

We can combine Eqs.5,6 and 8 to get

$$
\begin{aligned}
& u-(r, s)=P(r, s) \exp _{+\infty}\left[j 2 k_{P} Z\left(1-r^{2} / f^{2}\right)^{1 / 2}\right] \\
& \cdot R(r / f)\left(\lambda_{R} f\right)^{2} \int_{-\infty} u+(-r, s) P(-r, s) d s
\end{aligned}
$$

where $k_{P}=k_{R}+j\left(\alpha_{D}+\alpha_{L}\right)$. The output voltage, $v$, of the transducer can be determined as a function of the reflector position, $Z$, by integrating the $u^{*}(r, s) u^{-(r, s)}$ product over the $r-s$ plane to obtain

$V(Z)=\int_{-\infty}^{+\infty}\left[\int_{-\infty}^{+\infty} u+(r, s) P(r, s) d s\right]^{2} R(r / f)$

\section{Experimental Results}

Measurements are made to observe $V(Z)$ as given by Eq.11. with a set-up in pulse-echo arrangement. The results for a straight edge in aluminum are plotted in Fig.2 for various edge angles. $V(Z)$ curves for $90^{\circ}$ edges in aluminum, copper and steel are shown in Fig.3, Fig.4 depicts the $V(Z)$ curves for half and full SAW wavelength step discontinuities in aluminum. All the curves are different from each other showing either material or topology dependence of reflection coefficient.

We have also calculated $V(Z)$ using Eq.11 numerically with $u^{+}(r, s)=1$. An angle independent reflection coefficient is assumed [10],[11] within the angle of coverage. The results of this calculation are given in Fig.5. Notice that the agreement between the calculated curves and the measurement results are not particularly good. We suspect that this is due to uniform illumination assumption and idealizations on the pupil function. Nevertheless, the general variation of curves agree with the experi- 


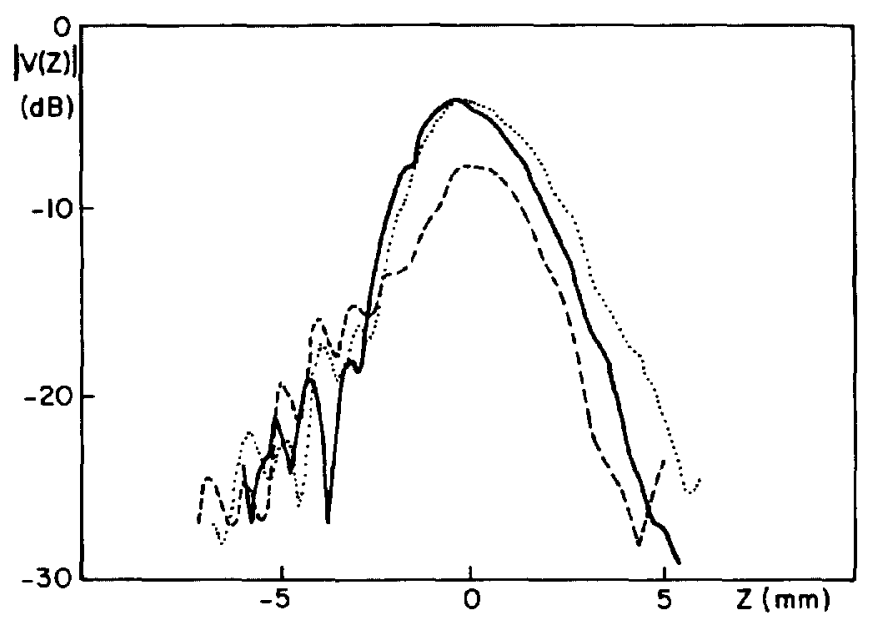

Fig.2 Measured $V(Z)$ curves for aluminum for $90^{\circ}$ (solid line), $40^{\circ}$ (dotted) and $30^{\circ}$ (dashed) edges.

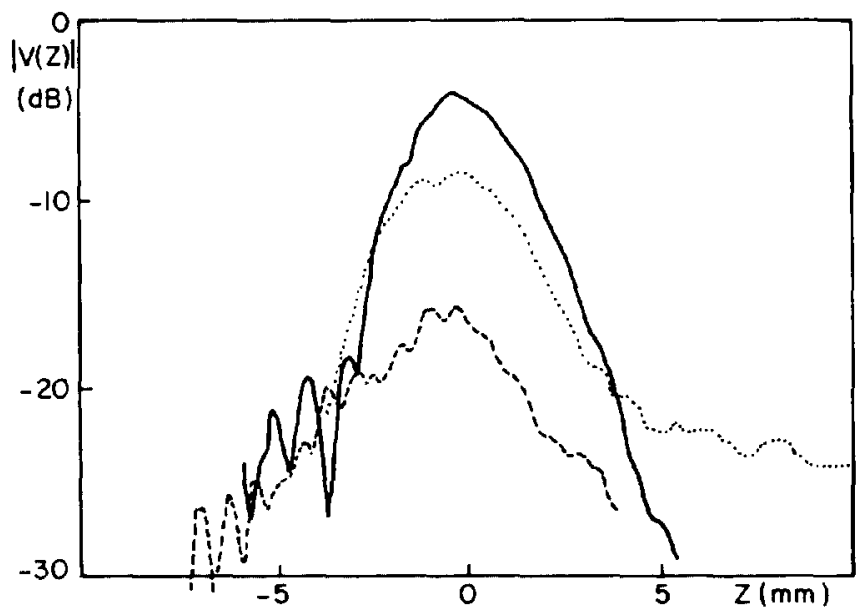

Fig. 3 Measured $V(Z)$ curves for $90^{\circ}$ edge on aluminum (solid line), steel (dotted) and copper (dashed).

mental results.

If it is possible to reverse the relation, one can obtain the reflection coefficient as a function of angle from the measured $V(Z)$ data, provided that the phase of $V(Z)$ can also be measured. Hildebrand et. al. [2] calculated the reflection coefficient at a liquid-solid interface from the measured $V(Z)$ data for a scanning acoustic microscope. We used a very similar approach to convert the $V(Z)$ relation in $E q .11$ with an approximation to a Fourier transform relation:

$R\left[\left(t-t^{2} / 4\right) 1 / 2\right] \pi((\tau-\tau / 2) / \tau m)=$ $j[(1-\tau / 2)(1-\tau / 4) 1 / 2] /(\operatorname{kgf} \sqrt{\tau})$

$\frac{1}{2 \pi \lambda} \int_{0}^{+\tau}\left[d / d Z\left(V(Z) \exp \left(-j k_{R} Z\right) / A(Z)\right)\right] \exp \left[j k_{R} Z \tau\right]$

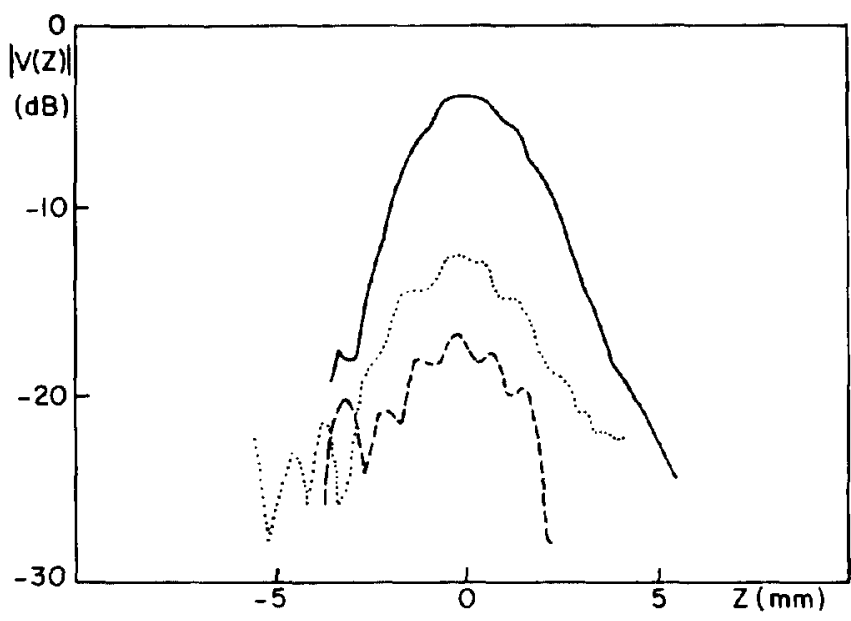

Fig. 4 Measured $V(Z)$ curves for $1 \mathrm{~mm}$ step down (solid line), $1 \mathrm{~mm}$ (dotted) and $2 \mathrm{~mm}$ (dashed) step ups in aluminum.

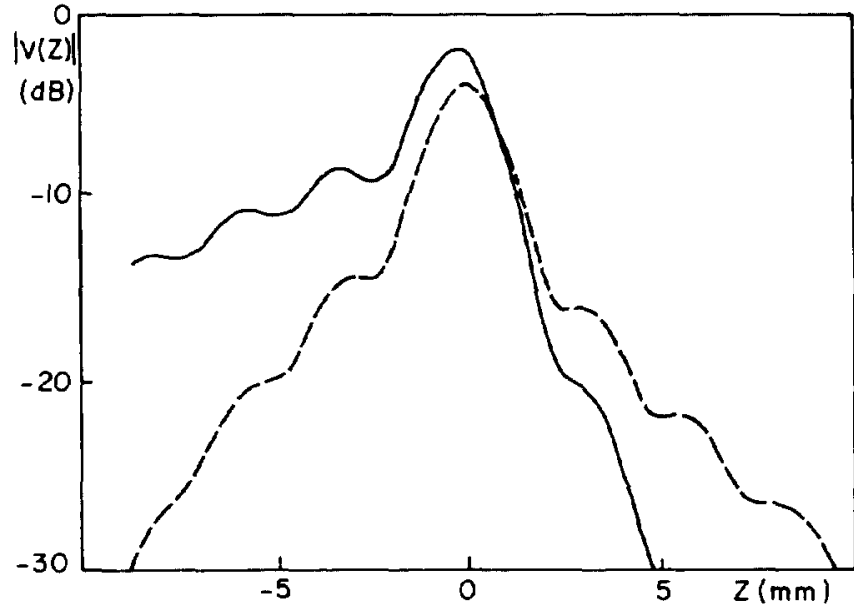

Fig.5 Calculated $V(Z)$ curves for aluminum (solid Iine) and steel (dotted).

where $\pi(x)=\left[\begin{array}{ll}1 & \text { if }|x|<1 / 2 \\ 0 & \text { otherwise }\end{array}\right.$,

$A(Z)=\left\{\exp \left[-\left(\alpha_{D}+\alpha_{L}\right) \min (0, Z)\right]\right.$

$\left.-\exp \left[-\left(\alpha_{D}+\alpha_{L}\right)(f+Z)\right]\right)^{2} /\left[\left(\alpha_{D}+\alpha_{L}\right)^{2} \tan ^{2} \theta_{R}\right]$, $\left(1-r^{2} / f^{2}\right)^{1 / 2}=1-\tau / 2$ and $\tau_{m}=2-2\left(1-x_{m^{2}} / f^{2}\right)^{1 / 2}$.

The reflected acoustic signal is digitized with a 6-bit accuracy at $25 \mathrm{MHz}$ and it is stored in a computer for every $Z$ value. It is then possible to eliminate the contribution of object independent background signal by a subtraction operation. The result can be Fourier transformed to obtain the phase sensitive $V(Z)$ data at a particular frequency. Having obtained the real and imaginary parts of $V(Z)$, the reflection coefficient is evaluated by using an FFT in the inversion integral. Notice that, the equally spaced FFT output would yield unequally spaced reflection coefficient values because of the nonlinear transforma- 
tion. The results of the calculation for an aluminum edge is plotted in Fig.6. A nonuniform reflection coefficient is obtained although no variation is expected within the angle of coverage [11].

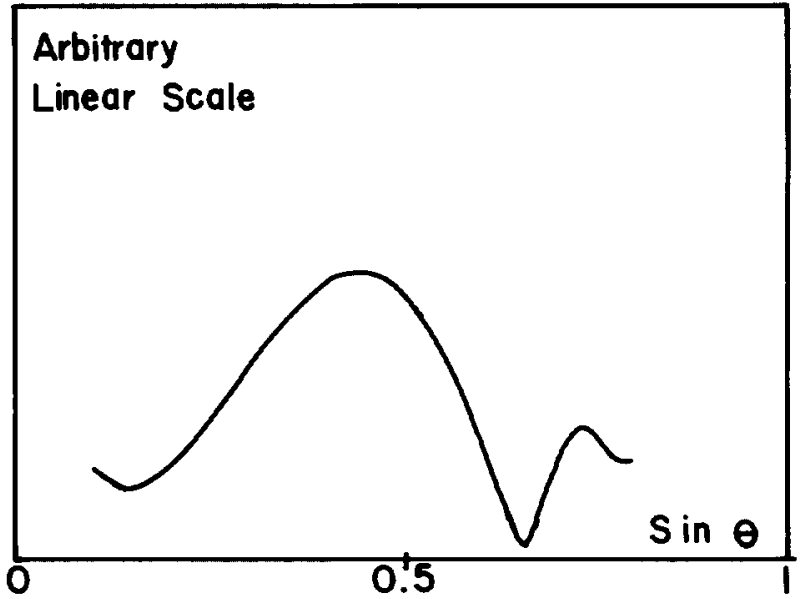

Fig.6 Reflection coefficient for $90^{\circ}$ edge of aluminum as calculated by the inversion of $V(Z)$.

\section{IV.Discussion}

The $V(Z)$ curves for SAWFAX have certain amount of material and topology dependence. However, this dependence is not as dramatic as in the case of bulk wave focusing lenses. The probable reason for this is relatively smooth nature of reflection coefficient. The function $R($.$) is quite$ independent of incidence angle within the angle of coverage [11]. $V(Z)$ curves are dependent mainly on the amplitude of the reflection coefficient. Consequently, the acoustic images obtained with such a focusing arrangement are easy to interpret and free from phase dependent artifacts. The reflected SAW amplitude variation with respect to edge angle and depth of cracks at normal incidence is reported in the literature $[12,13]$. Combining this information along with the SAWFAX images, it may be possible to obtain quantitative evaluation.

The inversion relation between the $V(Z)$ and the reflection coefficient is an ill-conditioned problem, due to its two-dimensional nature. It is very difficult to obtain reflection coefficient from measured $V(Z)$ data in the presence of any type of errors such as quantization error or measurement noise.

\section{References}

1. Special Issue of IEEE Son.Ultrason., vol. 32, No:2, 1985 .

2. J.A.Hildebrand, K.Liang and S.D.Bennett "Fourier transform approach to materials characterization with the acoustic microscope," J.Appl.Phys.,vol. 54, 7016-
7019,1983 .

3. K.K.Liang, G.S.Kino, and B.T.KhuriYakub, "Material characterization by the inversion of $\mathrm{V}(\mathrm{z})$," IEEE Trans. Son. Ultrason. vol. 32, pp. 213-224, 1985.

4. W.Parmon, and H.L.Bertoni, "Ray interpretation of the material signature in the acoustic microscope," Elect. Lett., vol. 15, pp. 685-686, 1979 .

5. H.Koymen, and A.Atalar, "Focusing surface waves using an axicon," Appl. Phys. Lett., vol. 47, pp. 1266-1268, 1985 .

6. A.Atalar, and H.Koymen, "Use of a conical axicon as a surface acoustic wave focusing device," IEEE Trans. Son. Ultrason. (to be published in January $1987)$.

7. A.Atalar, "An angular spectrum approach to contrast in reflection acoustic microscopy," J.Appl.Phys., vol 49, pp.51305139,1978 .

8. J.W.Goodman Fourier Optics McGraw-Hill, New York, 1968 .

9. H.L.Bertoni "Ray-optical evaluation of $V(Z)$ in the reflection acoustic microscope," IEEE Trans. Son. Ultrason. vol. $31, \mathrm{pp} .105-116,1984$.

10.F.C.Cuozzo, E.L.Cambiaggio, J-P Damiano, and E.Rivier, "Influence of elastic properties on Rayleigh wave scattering by normal discontinuities," IEEE Trans. on Son. Ultrason. vol. 24, pp.280-289, 1977 .

11.A.K.Gautesen, "Scattering of an obliquely incident Rayleigh wave in an elastic quarterspace," Wave Motion, vol. 8, pp. $27-41,1986$.

12.B.Q.Vu and V.K.Kinra "Diffraction of Rayleigh waves in a half-space. I. Normal edge crack," J. Acoust. Soc. Am., vol. 77, pp. 1425-1430, 1985.

13.V.K.Kinra and B.Q.Vu "Diffraction of Rayleigh waves in a half-space. II. Inclined edge crack," J. Acoust. Soc. Am., vol. 79, pp. 1688-1692, 1986. 